Courrier du Centre international Blaise Pascal

16 | 1994

Varia

\title{
Propositions sur le « Pari » de Pascal
}

\section{Laurent Thirouin}

\section{OpenEdition \\ Journals}

Édition électronique

URL : http://journals.openedition.org/ccibp/591

DOI : 10.4000/ccibp. 591

ISSN : 2493-7460

\section{Éditeur}

Centre international Blaise Pascal

\section{Édition imprimée}

Date de publication : 5 avril 1994

Pagination : 25-29

ISSN : 0249-6674

\section{Référence électronique}

Laurent Thirouin, «Propositions sur le « Pari » de Pascal », Courrier du Centre international Blaise Pascal [En ligne], 16| 1994, mis en ligne le 07 janvier 2016, consulté le 19 avril 2019. URL : http:// journals.openedition.org/ccibp/591 ; DOI : 10.4000/ccibp.591

Ce document a été généré automatiquement le 19 avril 2019.

Centre international Blaise Pascal 


\title{
Propositions sur le « Pari » de Pascal
}

\author{
Laurent Thirouin
}

1 Dans une heureuse formule, Philippe Sellier qualifie Pascal d'« Isaïe géomètre ». Après qu'il nous a montré la dimension prophétique des Pensées, dans leurs références comme dans leur écriture, il m'appartient de mettre en relief le caractère géométrique de l'œuvre, et ceci à travers un texte qui exhibe de façon hypertrophiée et quasi caricaturale la référence scientifique de son auteur: le célèbre argument connu sous le nom du «Pari ». L'usage des guillemets dans le titre de cette communication est intentionnel; il signale la violence faite au fr. 680 , dont on limite et fausse la portée, en réduisant l'argument à un de ses aspects seulement, le plus provocateur peut-être, mais certainement pas le plus important. Le pari n'est qu'un élément, un moment du complexe fr. 680 , que l'on serait mieux venu de désigner, soit avec neutralité par ses premiers mots « infini rien », soit avec plus d'audace, mais sans manquer de raisons, par l'expression que Pascal utilise à plusieurs reprises pour s'y référer, le Discours de la machine.

2 Les réflexions qui suivent n'ajoutent aucun élément vraiment nouveau au travail d'interprétation que l'on trouve dans mon étude sur le modèle du jeu dans la pensée de Pascal $^{1}$. J'essaierai simplement, dans un but pédagogique, de systématiser les points essentiels, en montrant notamment combien il est important d'identifier avec exactitude le substrat mathématique de l'argumentation, comment les travaux de Pascal sur les «partis » sont la clef indispensable pour comprendre non seulement la tonalité de son raisonnement, mais sa logique exacte, le sens de certains termes, la fonction même de la métaphore du jeu qui s'y déploie.

Le fragment du «Pari » a suscité un nombre étonnant de commentaires et de prises de position (adhésions plus ou moins naïves, rejets outrés). L'attention considérable portée à ce texte est en proportion directe du malaise qu'il produit. Et je verrais volontiers pour ma part une cause de ce malaise dans le caractère autophage du fr. 680 : entendons par là son obstination paradoxale à détruire lui-même ses propres élaborations conceptuelles et ce qui semblait d'abord constituer ses choix stratégiques :

1. La mathématisation

2. La notion de parti 

3. Le pari
4. Le parti-pris d'efficace.

Toutes ces notions, dont on montrera l'importance, qui font la teneur du raisonnement de Pascal, sont au bout du compte d'une troublante fragilité. Ainsi, découvrir un rouage de cet argument, c'est toujours aussi finir par découvrir le dysfonctionnement de ce rouage, que l'argument lui-même semble s'être donné pour mission de démontrer! D'où la grande difficulté de démonter ce texte, et le risque, au terme de sa quête, quand tous les éléments sont disjoints, de n'inventorier qu'un ensemble de pièces inefficaces.

5 Pour ne pas égarer mon auditoire dans ce jeu complexe et incertain, je me contenterai donc d'énoncer, sur un ton inévitablement dogmatique, quelques principes essentiels d'interprétation que le lecteur du fragment «infini rien» devrait respecter. On ne s'étonnera pas si le janséniste qui sommeille en tout pascalien a isolé à cet effet cinq propositions.

\section{Le recours aux probabilités?}

6 C'est une idée banale et sommaire, présente dans tout commentaire sur le "pari », que cette page de Pascal doit être mise en regard avec les travaux du géomètre sur le hasard, avec cette science naissante dont Pascal est indéniablement un fondateur, et qu'il baptisait lui-même avec jubilation du titre d'Aleae geometria ${ }^{2}$. Le fr. 680 - la chose est évidente - a des affinités avec les réflexions de Pascal sur les partis, c'est-à-dire la correspondance avec Fermat de l'année 1654 et les textes de la même année sur les usages du triangle arithmétique ${ }^{3}$. On retrouve dans ces pages des Pensées le vocabulaire caractéristique : les partis, les hasards de gain et de perte; la situation de jeu elle-même évoque le problème du joueur Méré, qui avait servi de prétexte au travail des mathématiciens.

7 Mais une fois signalée cette parenté, le problème reste entier : en quoi précisément les développements $d u$ «Pari» sont-ils tributaires des réflexions sur les partis? S'il s'agit simplement d'une convergence anecdotique, la chose a bien peu d'intérêt. On remarquera que l'apprenti théologien ne perd pas l'occasion de revenir sur ses domaines d'incontestable compétence. À moins que, comme certains critiques ${ }^{4}$, on ne l'accuse de mettre à profit son prestige scientifique, pour tenter, non sans une certaine malhonnêteté, d'impressionner l'honnête lecteur, en l'écrasant sous des références mathématiques qui le dépassent. L'appareil géométrique du texte ne serait déployé qu'à des fins principalement rhétoriques.

8 À cette inquiétude parfaitement légitime, on répondra par une mise en garde préalable, par quelques remarques plus ou moins évidentes qui touchent au statut des probabilités dans ce fr. 680. Il faut d'abord se garder d'une approche sommaire de l'histoire des sciences qui consiste à faire de Pascal l'inventeur du calcul des probabilités. Les spécialistes de cette question ${ }^{5}$ ont souligné depuis longtemps (mais leur voix ne porte pas toujours jusqu'aux critiques littéraires) que les positions de Pascal dans sa correspondance avec Fermat et ses développements sur les usages du triangle arithmétique ne recoupent que très marginalement la science des probabilités encore à naître. Est notamment absente de la réflexion pascalienne l'idée explicite d'un rapport entre les cas favorables et les cas possibles, en quoi consiste la définition élémentaire de la probabilité. En découvrant que les phénomènes aléatoires ressortissaient eux aussi au 
pouvoir du géomètre, Pascal faisait franchir un grand pas aux mathématiques, et il n'est pas déraisonnable, en ce sens, de célébrer en lui le fondateur de la géométrie du hasard. Mais les travaux concrets qu'il a menés en ce domaine s'apparentent bien davantage aux futures théories de la décision, développées à notre siècle, qu'à la science des probabilités, même sous une forme naissante. Une précaution essentielle en conséquence sera de ne pas transcrire les raisonnements mathématiques de Pascal sous la forme de nos habituelles équations : la tentation s'est toujours révélée forte chez les exégètes du fr. 680 et porte la responsabilité de nombreux faux-sens, ou tout au moins d'une perspective faussée.

9 Plus simple sans doute et plus élémentaire est la mise en garde suivante. Elle n'en reste pas moins primordiale. Frappé par le caractère apparemment démonstratif du texte, le lecteur superficiel risque en effet de le ranger dans l'arsenal des preuves apologétiques. Il doit rester bien clair que le fr. 680 ne propose à aucun moment une preuve mathématique de l'existence de Dieu. Il repose à l'inverse sur la résignation à n'avoir aucune aide de la raison dans la quête de Dieu. Le "pari» ne cherche pas à prouver que Dieu existe, puisqu'il intervient quand on a renoncé à le prouver. Mais il ne cherche pas davantage à fixer les probabilités de son existence : il ne prétend sur ce point encore à aucun résultat. Il se contente de passer en revue toutes les conditions possibles du problème, sans chercher à en privilégier aucune. La règle des partis doit bien être identifiée pour ce qu'elle est, une règle d'action, qui ne vise en aucune façon, même indirectement, à faire progresser la connaissance.

\section{L'interruption}

10 Une étrange formule du texte exige d'être élucidée : "cela ôte tout parti ». Outre son obscurité, l'expression devrait d'autant plus retenir l'exégète qu'elle concerne la notion clef de la géométrie du hasard. Le contexte éclaire certes le lecteur.

Cela ôte tout parti : partout où est l'infini et où il n'y a pas infinité de hasards de perte contre celui de gain, il n'y a point à balancer, il faut tout donner.

Dans une première rédaction, Pascal insistait dans les mêmes termes : «il n'y a point de parti », avant de corriger cette répétition en la remplaçant par le texte définitif : « il n’y a point à balancer ». L'absence de parti équivaut donc clairement à la disparition de toute hésitation. Les annotateurs les plus scrupuleux soulignent généralement cette équivalence, mais se gardent bien d'expliquer comment le terme de parti - qui signifie éventuellement « décision » - peut prendre ici le sens diamétralement opposé d'indécision. Comment ôter tout parti a-t-il chez Pascal le sens de forcer une décision? Il s'agit donc moins ici de trouver le sens de la phrase que de comprendre comment l'expression utilisée par l'auteur lui donne le sens que l'on a deviné. Le problème semblera à certains d'une pointilleuse érudition philologique. À tort, car il concentre toute la logique de l'argumentation pascalienne. Le terme de parti renvoie à une situation concrète (le problème posé par le joueur Méré), qui oriente donc très précisément un usage métaphorique. On ne comprendra pas la comparaison sur laquelle repose le fr. 680 , si l'on ne se fait pas une idée du comparant autant que du comparé. Le terme de parti suggère enfin une rationalité mathématique spécifique, qu'il est indispensable de préciser.

Essayons donc de répondre aussi clairement que possible à cette question: qu'est-ce qu'un parti ? Il est à noter d'abord que Pascal aurait pu utiliser le terme de " probabilité ", que l'on rencontre dans la Logique de Port-Royal, dans des pages dont il est sans nul doute 
l'inspirateur ${ }^{6}$. Mais pour lui, le terme évoquait vraisemblablement l'appareil intellectuel des jésuites. II ne faut certes pas confondre la notion jésuite de probabilité et le sens que prend aujourd'hui le même terme. Mais par delà les différences, la probabilité des jésuites peut se définir comme une théorie d'action face à l'incertitude : que conclure et qui suivre quand plusieurs opinions s'affrontent? Le parti pascalien est ainsi, d'une certaine manière, une alternative à la probabilité jésuite. Tandis que celle-ci aboutit à donner au sujet embarrassé toute latitude d'action, celui-là s'efforce de déterminer le seul comportement rationnellement satisfaisant, et par voie de conséquence contraignant.

Deux sèmes appartiennent intimement à la notion de parti : celui d'interruption et celui d' équilibre. Le parti s'inscrit dans un processus aléatoire qui n'a pu suivre son cours. Les joueurs de Méré font appel au mathématicien pour répartir les mises, dans un jeu de hasard qu'ils ont dû interrompre avant son terme naturel. C'est de l'interruption que provient la réémergence de la rationalité. Si les joueurs avaient pu poursuivre leur activité jusqu'à la victoire de l'un d'entre eux, le hasard aurait régné en maître définitivement. Quant au travail du mathématicien, il aboutit à déterminer un point d'équilibre (non pas une donnée numérique, mais un ensemble de valeurs numériques): en calculant le parti du jeu, Pascal établissait pour Méré une égalité artificielle entre le risque et la possession (une certaine possession); il reconstituait une situation remarquable, où il serait exactement équivalent pour un joueur de quitter le jeu en possession d'une certaine somme, ou de continuer à tenter le hasard pour en obtenir, éventuellement, une supérieure. Ma définition sera donc la suivante. Le parti est, si l'on s'en tient au problème de Méré, un partage provoqué par l'interruption d'une activité, mais un partage parfaitement juste, qui fait correspondre avec exactitude hasard et possession.

On le voit, le jeu qui sert implicitement de modèle à l'argumentation du fr. 680 est un jeu entamé, un jeu qu'il s'agit éventuellement d'interrompre. La situation existentielle de l'homme correspondant aux termes des partis n'est donc pas exactement celle de quelqu'un qui hésite à parier ou non, mais de celui qui hésite à interrompre ou non un jeu qui est en train de se dérouler, à «renoncer à l'attente du hasard et rentrer [...] en la propriété de quelque chose ${ }^{7}$ ». Si le géomètre était capable ici, comme pour Méré, de calculer un parti, il mettrait son interlocuteur dans une situation de choix équitable, il le placerait devant une alternative dont il serait également raisonnable de suivre chaque branche. Mais précisément, il n'y a pas ici de parti ; la situation, telle qu'elle a été définie, " ôte tout parti ». Le géomètre ne peut calculer aucun équivalent dont la possession compenserait exactement l'abandon des espérances aléatoires. S'il n'y a pas de parti, au sens mathématique du terme, le joueur n'a pas d'autre comportement raisonnable que de continuer à jouer.

\section{Le pari : argument a fortiori ; le pari : argument transitoire}

Pour donner le plus de force à son argument, Pascal passe par le détour des conditions qui lui sont le plus défavorables. Il accepte d'abord que les probabilités que Dieu existe soient infimes, c'est-à-dire qu'il y ait un seul hasard de gain face à un nombre fini, mais considérable, de hasards de perte. Il concède ensuite que la mise risquée est d'une grande valeur. Aucune de ces deux hypothèses n'est en fait avérée. Mais, si le raisonnement fondé sur la règle des partis est tenable dans cette situation, combien plus fort deviendra-t-il si les hypothèses de travail viennent à être remises en question. Or c'est ce 
qui se produira dans une optique chrétienne, sur laquelle l'auteur ne pouvait bien sûr pas faire fond dans un premier temps. Pour le chrétien en effet, la probabilité que Dieu existe touche, par la Foi, à la certitude; et la mise que représente la vie terrestre, abandonnée en apparence dans l'acceptation du pari, se révèle en réalité une mise sans valeur.

Que me promettez-vous enfin? car dix ans est le parti, sinon dix ans

d'amour-propre, à bien essayer de plaire sans y réussir, outre les peines certaines?

(fr. 153)

Cette réflexion, tirée de la liasse "Commencement", jette une nouvelle lumière sur le parti, c'est-à-dire sur le bien que je récupérerai si je renonce à jouer. Les dix ans d'existence que l'on peut, compte tenu des risques d'accident et de maladie, tenir statistiquement pour assurés, ne sont pas des années de plaisir. Le prix attribué par l'incroyant à sa propre vie - évaluation de la mise que retenait l'apologiste sans discuter tient en fait à une double illusion : sur la durée et sur la qualité. La foi chrétienne, si l'on pouvait recourir à elle, aurait pour effet immédiat de dissiper cette illusion.

Ainsi, si les données initiales du fr. 680 étaient les seules acceptables par un incroyant, elles se trouvent fondamentalement modifiées à la fin de l'argumentation. En supposant que le raisonnement de l'apologiste ait pu conduire l'incroyant à la foi, ce dernier en viendrait fatalement à la conclusion que le risque était nul et qu'il n'a rien parié. L'argument dit « du pari » débouche de la sorte sur deux enseignements déconcertants :

- qu'il est impossible de parier (nous allons aussitôt revenir sur ce point);

- qu'il n'y a pas de pari (qu'en matière de foi, la métaphore du pari n'est pas tenable).

Le modèle du « pari » que semble adopter le fr. 680 est donc bien un modèle transitoire, justifié par des nécessités rhétoriques, mais promis à la destruction.

\section{Place du pari dans le fragment « infini rien »}

19 Il est temps de justifier les guillemets imposés jusqu'ici au terme de pari, la réticence que nous avons manifestée devant l'appellation traditionnelle de ce fr.680. Le moment du pari doit être replacé en effet dans la scène intégrale qui se joue entre l'apologiste et son interlocuteur. Notre texte, si on le considère dans son ensemble, sans se limiter à la provocante partie mathématique, se présente comme une lente retraite rhétorique, qui se conclut sur une fin de non-recevoir parfaitement malhonnête. La démonstration progresse au rythme des reculades de l'interlocuteur, d'une cascade de « mais » au terme de laquelle, forcé dans ses derniers retranchements, le raisonneur malheureux choisit tout simplement de balayer le raisonnement d'un revers de main.

20 L'apologiste entame le débat par une défense de la position chrétienne. Les chrétiens ne revendiquent pas un fondement rationnel à leur croyance. On n'est donc pas en droit de le leur reprocher.

- Oui, mais encore que cela excuse ceux qui l'offrent telle, et que cela les ôte du

blâme de la produire sans raison, cela n'excuse pas ceux qui la reçoivent.

21 Privé irrémédiablement du secours de la raison, rétorque l'apologiste, un choix peut légitimement se fonder sur des critères de substitution. On ne doit pas le critiquer pour cela.

- Non mais je les blâmerai d'avoir fait non ce choix, mais un choix [...] Le juste est de ne point parier. 

les deux termes de ce pari.

- Oui il faut gager, mais je gage peut-être trop.

Voilà l'objection qui provoque le long développement mathématique, et sa triomphale conclusion enfin, donnée pour irrécusable: «si les hommes sont capables de quelque vérité, celle-là l'est ».

- Je le confesse, je l'avoue, mais encore n'y a-t-il point moyen de voir le dessous du jeu?

La demande même de l'incroyant, qui déplace une nouvelle fois le problème, sans opposer à la démonstration précédente quelque objection que ce soit, manifeste une troublante résistance, une première transgression $\mathrm{du}$ contrat tacite sur lequel repose une controverse. L'apologiste prend acte de ce déplacement et abandonne la logique argumentative dans laquelle il s'inscrivait. "L'Écriture et le reste », qu'il évoque alors, représentent bel et bien ici le dessous du jeu: des éléments hétérogènes, susceptibles de renforcer une conviction acquise par d'autres voies.

- Oui mais j'ai les mains liées [...] Et je suis fait d'une telle sorte que je ne puis croire.

À cette ultime marque de mauvaise foi, à cette façon brutale de révoquer tous les acquis du débat, on comprendrait que l'apologiste réagisse par l'exaspération. Il n'en sera rien. L'incroyant en fait se comporte ici selon la droite logique étudiée par Pascal dans son Art de persuader, "qui consiste autant en celui d'agréer qu'en celui de convaincre ${ }^{8}$ ». Les raisons démonstratives font une fois de plus la preuve de leur inaptitude à persuader un homme dont la volonté détermine en réalité toutes les croyances. Le géomètre du fr. 680 ne se donnera donc pas le ridicule de faire valoir le long travail démonstratif qu'il a mené à bien, de représenter à son interlocuteur l'inconséquence intellectuelle dont il fait preuve. L'apologiste accepte, sans ébaucher la moindre résistance, de voir toute son argumentation réduite à néant.

Il est vrai, mais apprenez au moins que votre impuissance à croire...

Le fr. 680 se développe à partir de là sur les ruines du "pari », sur l'inefficacité d'un raisonnement, rationnellement contraignant mais incapable de forcer l'adhésion. Le recours à la règle des partis apparaît ainsi doublement malheureux. Techniquement d'abord, parce que la méthode mise au point par Pascal et Fermat ne s'applique pas dans ce contexte : «Cela ôte tout parti ». La situation existentielle de l'homme, entre infini et rien, ne permet pas d'établir un parti. L'aide du géomètre, spécialiste en calcul de partis, est donc inutile : après avoir été exhibé de façon spectaculaire, le calcul est congédié. Rhétoriquement ensuite, parce que l'interlocuteur n'est pas entraîné par la démonstration. S'il doit tirer une conclusion pratique de l'argument, c'est en méditant sur l'inefficacité de cet argument, en prenant conscience que, contrairement à son sentiment initial, il a fait un choix, et que - plus grave encore- ce choix est déraisonnable.

\section{Les conditions du problème sont (de façon subreptice) l'enjeu réel de l'argument}

Renonçant d'une certaine manière à faire parier l'incroyant pour Dieu, le fr. 680 obtient peut-être son effet principal sur un plan psychologique : il donne le sentiment que l'on a parié; il installe l'homme dans l'obligation de jouer. Un problème logique apparait 
néanmoins ici, et une ambiguïté dans les termes qu'il nous faut réduire. Quand un joueur est par hypothèse contraint à jouer, comme le répète inlassablement l'apologiste à chaque stade de l'argumentation, quelle peut être la nature des conseils qu'on lui donne? Le raisonnement du géomètre semble parfois déboucher sur une tautologie assez vaine. On attend qu'il nous expose la meilleure façon de jouer, or le principal aboutissement de son travail semble être le seul conseil de jouer. Si on écarte les variables de la démonstration, la conclusion, réduite à sa seule armature, parait presque impertinente : « vous agiriez de mauvais sens, étant obligé à jouer, de refuser de jouer... ». Pascal veut-il convaincre l'incroyant de participer à un jeu auquel il est, de toute façon, forcé de participer? Pratiquement le conseil est inutile. Peut-être se place-t-il sur un plan théorique et cherche-t-il à justifier la contrainte que subit le joueur, en lui prouvant qu'elle n'est pas désavantageuse. Le projet serait sans grand intérêt et surtout peu conforme à la préoccupation dont témoigne tout le discours, de définir une ligne d'action. Que signifie concrètement l'obligation de jouer et quelle latitude laisse-t-elle à l'homme?

L'apparent illogisme vient de ce que le verbe jouer est pris dans deux acceptions différentes. Quand il est employé transitivement, comme dans l'expression «jouer une vie contre trois ", il est synonyme de gager ("vous pourriez encore gager ») ou hasarder ("vous seriez imprudent [...] de ne pas hasarder votre vie »). Il désigne alors le choix précis de jeu qui consiste à parier pour l'existence de Dieu. Le choix inverse, c'est-à-dire le pari contre Dieu, n'est pas nommé spécifiquement, mais il se confond avec le refus du jeu. À partir du moment où le pari pour Dieu est jugé le seul comportement raisonnable ("gagez donc qu'il est sans hésiter»), l'unique question qui se pose est celle de la grandeur de la mise (« je gage peut-être trop ») et le verbe gager se réfère exclusivement à l'un des deux paris théoriquement possibles («vous pourriez encore gager », "vous auriez encore raison de gager un pour avoir deux»). Pascal refuse d'envisager un pari contre Dieu, il s'abstient même d'une formulation tellement logique que les éditeurs de Port-Royal ne résisteront pas à l'ajouter : « ne parier point que Dieu est, c'est parier qu'il n'est pas ». Il faut s'interroger sur l'absence de cette formule dans le texte pascalien. Observons seulement pour l'instant que Pascal s'en tient à une définition restreinte du jeu : seuls jouent finalement ceux qui ont parié pour Dieu, les autres sont considérés comme ayant arrêté de jouer.

Contrairement aux apparences, le propos du fr. 680 n'est pas de déterminer s'il faut ou non parier pour Dieu. Le jeu étant déjà en cours, il ne s'agit pas de savoir si l'on va ou non aller vers Dieu, mais plutôt si l'on va se soustraire à Dieu. Sur un plan psychologique, la différence est énorme. Arrêter le jeu avant son interruption fatale (la mort) - devancer la fin - c'est l'équivalent d'un suicide : à l'inverse de ce que l'on aurait supposé, c'est le non joueur qui fait preuve d'imprudence. Un autre repentir de Pascal dénote ici clairement le mouvement de sa pensée. «Il faut renoncer à la raison pour choisir la vie plutôt que de la hasarder... ", concluait-il dans un premier temps. L'auteur remplace le verbe choisir par le verbe garder ("garder la vie plutôt que de la hasarder ») qui éclaire d'une tout autre façon l'attitude de l'incroyant endurci : ce n'est pas quelqu'un qui agit positivement, qui exerce une faculté de choix, mais un joueur qui refuse le jeu, qui préfère garder. L'inconséquence de cette position (être joueur et ne pas jouer) est le fondement de l'imprudence que dénonce Pascal.

Du modèle initial du pari (" deux choses à engager », « je gage peut-être trop ») on a glissé à celui des partis: l'interruption d'un processus aléatoire. Par l'argument pascalien, l'homme se trouve mis dans la nécessité non pas d'agir, mais de réagir. C'est cette 
situation tout à fait particulière (les simples données du problème en apparence) qui fait le cœur même du discours. En réalité, l'efficace de l'argument du pari ne réside pas dans sa conclusion, mais dans l'imposition subreptice de ses données de départ. On comprend mieux alors, si le but de l'apologiste est d'insinuer chez son interlocuteur le sentiment qu'il prend déjà part à un jeu, pourquoi Pascal évite de proposer une brutale application théologique de la notion d'espérance mathématique; pourquoi à chaque étape du raisonnement est répétée avec une telle constance l'obligation de jouer. On n'a pas suffisamment vu le sens mathématique de cette obligation de jouer, qui fait la subtile différence entre un problème d'espérance mathématique et un problème de partis 9 . Mais la signification religieuse de ce modèle mathématique est le point essentiel : ce qui est implicitement posé dans les données du problème, c'est l'appel divin, un appel tellement précieux qu'il dépasse toute équivalence.

Que l'on se demande en effet pourquoi le calcul des partis, qui était possible dans le problème de Méré, est ici exclu.

Pour entendre les règles des partis, la première chose qu'il faut considérer est que l'argent que les joueurs ont mis au jeu ne leur appartient plus, car ils en ont quitté la propriété ; mais ils ont reçu en revanche le droit d'attendre ce que le hasard leur en peut donner, suivant les conditions dont ils sont convenus d'abord.

Mais comme c'est une loi volontaire, ils la peuvent rompre de gré à gré ; et ainsi, en quelque terme que le jeu se trouve, ils peuvent le quitter; et, au contraire de ce qu'ils ont fait en y entrant, renoncer à l'attente du hasard, et rentrer chacun en la propriété de quelque chose. Et en ce cas le règlement de ce qui doit leur appartenir doit être tellement proportionné à ce qu'ils avaient droit d'espérer de la fortune que chacun d'eux trouve entièrement égal de prendre ce qu'on lui assigne ou de continuer l'aventure du jeu; et cette juste distribution s'appelle le parti ${ }^{10}$.

On lit mal le fragment «Infini rien » si l'on pense que l'argument mathématique répond à la question «faut-il parier pour Dieu? ». Le problème se pose plutôt en ces termes : qui interrompra le jeu, Dieu ou l'homme? C'est une erreur de considérer la vie terrestre comme une mise, dont l'homme aurait la libre disposition, qu'il choisirait ou non de risquer : la mise échappe à l'homme. Quand il naît elle est déjà faite, les conditions du jeu sont arrêtées. La vie terrestre est en réalité le parti, l'avoir que peut récupérer l'homme s'il préfère arrêter le jeu. On s'explique ainsi le ton catégorique de Pascal : «vous seriez imprudent lorsque vous êtes forcé à jouer de ne pas hasarder votre vie pour en gagner trois à un jeu où il y a pareil hasard de perte et de gain ». Décider de ne pas hasarder alors même qu'on joue (puisqu'on est «forcé à jouer »), cela correspond à interrompre le jeu (et non pas à s'en abstenir). Ce jeu, favorable, met l'homme dans une situation qu'il n'a pas méritée (achetée) mais héritée de par sa condition d'homme. Refuser cette condition c'est accepter de régresser, échanger un privilège contre un statut misérable. Si le privilège était grand, c'est une imprudence ; s'il était infini, c'est une folie.

Quand on est forcé à jouer, il faut renoncer à la raison, pour garder la vie plutôt que de la hasarder pour le gain infini...

Ce que l'argument du discours laisse entrevoir, derrière le rideau de fumée des développements mathématiques, c'est la présence de la Grâce. 


\section{NOTES}

1. L. Thirouin, Le Hasard et les règles: le modèle du jeu dans la pensée de Pascal. Paris: Vrin, 1991 (222 p.).

2. Celeberrimae matheseos Academiae Parisiensis (1654), CEuvres complètes, éd. J. Mesnard, t. II, p. 1034.

3. Pascal, Euvres complètes, éd. J. Mesnard, t. II, p. 1132 sq., p. 1166 sq.

4. V. Carraud, Pascal et la philosophie, P.U.F., 1992, p. 439.

5. Voir par exemple les travaux d'E. Coumet, et notamment son étude : «La théorie du hasard est-elle née par hasard ?", Annales (Économie Sociétés Civilisations), n 3, mai-juin 1970, pp. 574-597. 6. Arnauld et Nicole, La Logique ou l'art de penser, $4^{\mathrm{e}}$ partie, chap. XVI : « Du jugement qu'on doit faire des accidents futurs ".

7. Usage du triangle arithmétique (đuvres complètes, éd. J. Mesnard, t. II, p. 1308).

8. Pascal, Euvres complètes, éd. J. Mesnard, t. III, p. 416.

9. Pour cette question, un peu technique, je renvoie à mon développement : « la valeur pratique du calcul des probabilités » (Le Hasard et les règles..., p. 147-157).

10. Usage du triangle arithmétique (Euvres complètes, éd. J. Mesnard, t. II, p. 1308 ).

INDEX

Mots-clés : Pascal, Pensées, pari

Keywords : wager

\section{AUTEUR}

\section{LAURENT THIROUIN}

GRAC, Institut d'Histoire de la Pensée Classique, Université Lumière Lyon 2. 